\title{
A proof of a conjecture of Marques and Trojovsky
}

\author{
Emrah Kiliç
}




\title{
A PROOF OF A CONJECTURE OF MARQUES AND TROJOVSKY
}

\author{
EMRAH KILIC
}

Received 28 June, 2012

\begin{abstract}
In this paper, we consider Marques and Trojovsky's conjecture involving Fibonomial coefficients, Fibonacci and Lucas numbers. After rewriting it in terms of Gaussian $q$-binomial coefficients and $q$-Pochhammer symbols, we give a solution of the conjecture. The approach is to use $q$-analysis to prove the claimed formulas.
\end{abstract}

2010 Mathematics Subject Classification: 11B39

Keywords: Fibonomial coefficients, Gaussian $q$-binomial coefficients, $q$-calculus

\section{INTRODUCTION}

The Fibonomial coefficient is, for $n \geq m \geq 1$, defined by

$$
\left\{\begin{array}{l}
n \\
m
\end{array}\right\}_{F}:=\frac{F_{1} F_{2} \ldots F_{n}}{\left(F_{1} F_{2} \ldots F_{n-m}\right)\left(F_{1} F_{2} \ldots F_{m}\right)}
$$

with $\left\{\begin{array}{l}n \\ n\end{array}\right\}=\left\{\begin{array}{l}n \\ 0\end{array}\right\}=1$ where $F_{n}$ is the $n$th Fibonacci number.

Recently as an interesting generalization of the binomial coefficients, the Fibonomial coefficients have taken the interest of several authors. For their properties, we refer to $[2,3,5]$.

The Gaussian $q$-binomial coefficient $\left[\begin{array}{c}n \\ m\end{array}\right]_{q}$ is defined, for all real $n$ and integers $m$ with $m \geq 0$, by

$$
\left[\begin{array}{c}
n \\
m
\end{array}\right]_{q}:=\frac{(q ; q)_{n}}{(q ; q)_{m}(q ; q)_{n-m}}
$$

and as zero otherwise, where the $q$-Pochhammer symbol is defined as

$$
(a ; q)_{n}=(1-a)(1-a q) \ldots\left(1-a q^{n-1}\right) .
$$

Thus, $\left[\begin{array}{c}n \\ m\end{array}\right]_{q}$ is a rational function of the parameter $q$ (for more details, see $[1,10.2 .2(\mathrm{c})]$ ). Let

$$
\alpha=\frac{1+\sqrt{5}}{2} \text { and } \beta=\frac{1-\sqrt{5}}{2} \text {. }
$$


Then the well known Binet forms give

$$
F_{n}=\frac{\alpha^{n}-\beta^{n}}{\alpha-\beta}, \quad L_{n}=\alpha^{n}+\beta^{n},
$$

and thus

$$
F_{n}=\alpha^{n-1} \frac{1-q^{n}}{1-q}, \quad L_{n}=\alpha^{n}\left(1+q^{n}\right),
$$

with $q=\beta / \alpha=-\alpha^{-2}$, so that $\mathbf{i}=\alpha \sqrt{q}$, where $F_{n}$ is the $n$th Fibonacci number and $L_{n}$ is the $n$th Lucas number.

All the identities we are going to prove hold for general $q$, and results about Fibonacci and Lucas numbers come out as corollaries for the special choice of $q$.

The link between the Fibonomial and Gaussian $q$-binomial coefficients is

$$
\left\{\begin{array}{l}
n \\
m
\end{array}\right\}_{F}=\alpha^{m(n-m)}\left[\begin{array}{l}
n \\
m
\end{array}\right]_{q} \text { with } q=-\alpha^{-2} .
$$

For the reader's convenience and later use, we recall Rothe's formula [1, 10.2.2(c)]:

$$
\sum_{k=0}^{n}\left[\begin{array}{l}
n \\
k
\end{array}\right]_{q}(-1)^{k} q^{\left(\begin{array}{c}
k \\
2
\end{array}\right)} x^{k}=(x ; q)_{n} .
$$

In a recent paper, Marques and Trojovsky [7] provided various sums including Fibonomial coefficients, Fibonacci and Lucas numbers (for various sums properties of the coefficients, we refer to [4,6]). For example, for positive integers $m$ and $n$, the authors [7] showed that

$$
\begin{gathered}
\sum_{j=0}^{4 m+2}(-1)^{\frac{j(j+1)}{2}}\left\{\begin{array}{c}
4 m+2 \\
j
\end{array}\right\}_{F} L_{2 m+1-j}=-\left\{\begin{array}{c}
4 m+2 \\
4 n+3
\end{array}\right\}_{F} \frac{F_{4 n+3}}{F_{2 m+1}}, \\
\sum_{j=0}^{4 m+2}(-1)^{\frac{j(j-1)}{2}}\left\{\begin{array}{c}
4 m \\
j
\end{array}\right\}_{F} L_{2 m-j}=-\left\{\begin{array}{c}
4 m \\
4 n+1
\end{array}\right\}_{F} \frac{F_{4 n+1}}{F_{2 m}}, \\
\sum_{j=0}^{4 m+2}(-1)^{\frac{j(j-1)}{2}}\left\{\begin{array}{c}
4 m \\
j
\end{array}\right\}_{F} F_{n+4 m-j}=\frac{1}{2} F_{2 m+n} \sum_{j=0}^{4 m}(-1)^{\frac{j(j-1)}{2}}\left\{\begin{array}{c}
4 m \\
j
\end{array}\right\}_{F} L_{2 m-j}, \\
\sum_{j=0}^{4 m+2}(-1)^{\frac{j(j+1)}{2}}\left\{\begin{array}{c}
4 m+2 \\
j
\end{array}\right\}_{F} F_{n+4 m+2-j}=\frac{1}{2} F_{2 m+n+1} \times \sum_{j=0}^{4 m+2}(-1)^{\frac{j(j+1)}{2}}\left\{\begin{array}{c}
4 m+2 \\
j
\end{array} L_{F} L_{2 m+1-j .}\right.
\end{gathered}
$$

They also proved that

$$
\sigma_{1}(n)+\sigma_{2}(n)-\sigma_{3}(n)-\sigma_{4}(n)=0,
$$


where

$$
\begin{aligned}
& \sigma_{1}(n)=\sum_{j=0}^{m}\left\{\begin{array}{c}
4 m \\
4 j
\end{array}\right\}_{F} F_{n+4 m-4 j}, \sigma_{2}(n)=\sum_{j=0}^{m-1}\left\{\begin{array}{c}
4 m \\
4 j+1
\end{array}\right\}_{F} F_{n+4 m-4 j-1}, \\
& \sigma_{3}(n)=\sum_{j=0}^{m-1}\left\{\begin{array}{c}
4 m \\
4 j+2
\end{array}\right\}_{F} F_{n+4 m-4 j-2}, \sigma_{4}(n)=\sum_{j=0}^{m-1}\left\{\begin{array}{c}
4 m \\
4 j+3
\end{array}\right\}_{F} F_{n+4 m-4 j-3} .
\end{aligned}
$$

Also they conjectured three complex sum-product formulas involving the Fibonomial coefficients, Fibonacci and Lucas numbers. We recall this conjecture:

Conjecture 1. Let $m, n$ be any positive integers. Then

$$
\begin{aligned}
& \text { i) } \sigma_{1}(n)+\sigma_{2}(n)=F_{4 m+n} \prod_{k=1}^{2 m-1} L_{2 k}, \\
& \text { ii) } \sigma_{1}(n)-\sigma_{3}(n)=(-1)^{m} F_{2 m+n} L_{2 m} \prod_{k=1}^{2 m-1} L_{k}^{2}, \\
& \text { ii i) } \sigma_{1}(n)-\sigma_{4}(n)=F_{n} \prod_{k=1}^{2 m-1} L_{2 k} .
\end{aligned}
$$

Finally by solving the system of linear equations in (1.1) and the Conjecture, they state their last result as

Corollary 1. Let $m, n$ be any positive integers. Then

$$
\begin{aligned}
\text { i) } \sigma_{1}(n) & =\frac{1}{2} F_{2 m+n} L_{2 m}\left((-1)^{m} \prod_{k=1}^{2 m-1} L_{k}^{2}+\prod_{k=1}^{2 m-1} L_{2 k}\right), \\
\text { ii) } \sigma_{2}(n) & =\frac{1}{2}\left((-1)^{m+1} F_{2 m+n} L_{2 m} \prod_{k=1}^{2 m-1} L_{k}^{2}+L_{2 m+n} F_{2 m} \prod_{k=1}^{2 m-1} L_{2 k}\right), \\
\text { iii) } \sigma_{3}(n) & =\frac{1}{2} F_{2 m+n} L_{2 m}\left((-1)^{m+1} \prod_{k=1}^{2 m-1} L_{k}^{2}+\prod_{k=1}^{2 m-1} L_{2 k}\right), \\
\text { iv) } \sigma_{4}(n) & =\frac{1}{2}\left((-1)^{m} F_{2 m+n} L_{2 m} \prod_{k=1}^{2 m-1} L_{k}^{2}+L_{2 m+n} F_{2 m} \prod_{k=1}^{2 m-1} L_{2 k}\right) .
\end{aligned}
$$

In this paper, we shall prove the results of Corollary 1 rather than the Conjecture because if one can prove the results of Corollary 1, then the results of Conjecture could be easily proved by some simple algebra.

The present paper is organized as follows: (i) Before the proof of Corollary, we will give some auxiliary Lemmas. (ii) We rewrite it via the Gaussian $q$-binomial 
coefficients and the $q$-Pochhammer symbol. (iii) Finally we give a solution for the formulas mentioned in (ii). The approach is to use $q$-analysis to prove the claimed formulas.

\section{THE COROLLARY IN TERMS OF GAUSSIAN $q$-BINOMIAL COEFFICIENTS}

In this section, before the Corollary 1 , we give some auxiliary Lemmas :

Lemma 1. For nonnegative integer $n$ and arbitrary integer $c$,

$$
\prod_{k=0}^{n}\left(1+q^{k+c+1}\right)=\frac{1+q^{n+c+1}}{1+q^{c}} \prod_{k=0}^{n}\left(1+q^{k+c}\right) .
$$

Proof. (Induction on $n$ ) If $n=0$, then the claim is easily seen follows from

$$
1+q^{k+c+1}=\frac{1+q^{n+c+1}}{1+q^{c}}\left(1+q^{c}\right) .
$$

Suppose that the claim is true for $n>0$. Then we show that the claim is true for $n+1$. Consider

$$
\prod_{k=0}^{n+1}\left(1+q^{k+c+1}\right)=\left(1+q^{n+c+2}\right) \prod_{k=0}^{n}\left(1+q^{k+c+1}\right),
$$

which, by the induction hypothesis, equals

$$
\begin{aligned}
\prod_{k=0}^{n+1}\left(1+q^{k+c+1}\right) & =\left(1+q^{n+c+2}\right) \frac{\left(1+q^{n+c+1}\right)}{\left(1+q^{c}\right)} \prod_{k=0}^{n}\left(1+q^{k+c}\right) \\
& =\frac{\left(1+q^{n+c+2}\right)}{\left(1+q^{c}\right)} \prod_{k=0}^{n+1}\left(1+q^{k+c}\right),
\end{aligned}
$$

as claimed.

By using the Lemma 1 and the definition of $q$-Pochhammer symbol, we have the following lemmas:

Lemma 2. For any positive integer $m$ and arbitrary integer $n$,

i)

$$
\begin{aligned}
q^{-2 m-\frac{n-1}{2}}\left(-q^{-2 m+1} ; q\right)_{4 m}- & q^{2 m+\frac{n+1}{2}}\left(-q^{-2 m} ; q\right)_{4 m} \\
& =2 q^{-\frac{1}{2}\left(n+4 m^{2}+2 m-1\right)}(-q ; q)_{2 m}^{2} \frac{\left(1-q^{n+2 m}\right)}{\left(1+q^{2 m}\right)}
\end{aligned}
$$

ii)

$$
q^{-2 m-\frac{n-1}{2}}\left(-\mathbf{i} q^{-2 m+1} ; q\right)_{4 m}+q^{-2 m-\frac{n-1}{2}}\left(\mathbf{i} q^{-2 m+1} ; q\right)_{4 m}
$$




$$
\begin{aligned}
& -q^{2 m+\frac{n+1}{2}}\left(-\mathbf{i} q^{-2 m} ; q\right)_{4 m}-q^{2 m+\frac{n+1}{2}}\left(\mathbf{i} q^{-2 m} ; q\right)_{4 m} \\
= & 2(-1)^{m} q^{-\frac{1}{2}\left(n+4 m^{2}+2 m-1\right)}\left(1+q^{2 m}\right)\left(1-q^{n+2 m}\right)\left(-q^{2} ; q^{2}\right)_{2 m-1} .
\end{aligned}
$$

Proof. To prove (i), we rewrite it as in the clear form

$$
\begin{aligned}
q^{-2 m-\frac{n-1}{2}} \prod_{k=0}^{4 m-1}\left(1+q^{k-2 m+1}\right) & -q^{2 m+\frac{n+1}{2}} \prod_{k=0}^{4 m-1}\left(1+q^{k-2 m}\right) \\
& =2 q^{-\frac{n+4 m^{2}+2 m-1}{2}} \frac{\left(1-q^{n+2 m}\right)}{\left(1+q^{2 m}\right)} \sum_{k=1}^{2 m}\left(1+q^{k}\right)^{2}
\end{aligned}
$$

or

$$
\begin{aligned}
q^{m(2 m-1)} \prod_{k=0}^{4 m-1}\left(1+q^{k-2 m+1}\right) & -q^{2 m^{2}+3 m+n} \prod_{k=0}^{4 m-1}\left(1+q^{k-2 m}\right) \\
& =2 \frac{\left(1-q^{n+2 m}\right)}{\left(1+q^{2 m}\right)} \sum_{k=1}^{2 m}\left(1+q^{k}\right)^{2}
\end{aligned}
$$

Now we shall consider the left hand side of the above equation:

$$
q^{m(2 m-1)} \prod_{k=0}^{4 m-1}\left(1+q^{k-2 m+1}\right)-q^{2 m^{2}+3 m+n} \prod_{k=0}^{4 m-1}\left(1+q^{k-2 m}\right) .
$$

By Lemma 1, we rewrite it as

$$
\begin{aligned}
& q^{m(2 m-1)}\left(\frac{1+q^{4 m-1-2 m+1}}{1+q^{-2 m}}\right) \prod_{k=0}^{4 m-1}\left(1+q^{k-2 m}\right) \\
& -q^{2 m^{2}+3 m+n} \prod_{k=0}^{4 m-1}\left(1+q^{k-2 m}\right) \\
& =\left(q^{m(2 m-1)}\left(\frac{1+q^{2 m}}{1+q^{-2 m}}\right)-q^{2 m^{2}+3 m+n}\right) \prod_{k=0}^{4 m-1}\left(1+q^{k-2 m}\right) \\
& =q^{m(2 m+1)}\left(1-q^{2 m+n}\right) \prod_{k=0}^{4 m-1}\left(1+q^{k-2 m}\right) .
\end{aligned}
$$

Since

$$
\prod_{k=0}^{4 m-1}\left(1+q^{k-2 m}\right)=\frac{2}{q^{m(2 m+1)}\left(1+q^{2 m}\right)} \sum_{k=1}^{2 m}\left(1+q^{k}\right)^{2},
$$


we get

$$
\begin{aligned}
& q^{m(2 m-1)} \prod_{k=0}^{4 m-1}\left(1+q^{k-2 m+1}\right)-q^{2 m^{2}+3 m+n} \prod_{k=0}^{4 m-1}\left(1+q^{k-2 m}\right) \\
& =\frac{2 q^{m(2 m+1)}\left(1-q^{2 m+n}\right)}{q^{m(2 m+1)}\left(1+q^{2 m}\right)} \sum_{k=1}^{2 m}\left(1+q^{k}\right)^{2} \\
& =2 \frac{\left(1-q^{2 m+n}\right)}{\left(1+q^{2 m}\right)}(-q ; q)_{2 m}^{2},
\end{aligned}
$$

as claimed.

The second claim is similarly obtained.

The Lemma 2 will be required to prove the first claim of Corollary 1 . We give the following Lemma to prove the second claim of Corollary 1 without proof:

Lemma 3. For any positive integer $m$ and arbitrary integer $n$,

i)

$$
\begin{aligned}
& q^{2 m-1}\left(-q^{-2 m+1} ; q\right)_{4 m}-q^{n+6 m-1}\left(-q^{-2 m} ; q\right)_{4 m} \\
& =\frac{2\left(1-q^{n+2 m}\right)\left(1+q^{2 m}\right)}{q^{(m-1)(2 m-1)}}(-q ; q)_{2 m-1}^{2}
\end{aligned}
$$

and

ii)

$$
\begin{aligned}
& \mathbf{i} q^{2 m-1}\left(\left(\mathbf{i} q^{-2 m+1} ; q\right)_{4 m}-\left(-\mathbf{i} q^{-2 m+1} ; q\right)_{4 m}\right) \\
& -\mathbf{i} q^{n+6 m-1}\left(\left(\mathbf{i} q^{-2 m} ; q\right)_{4 m}-\left(-\mathbf{i} q^{-2 m} ; q\right)_{4 m}\right) \\
& =2(-1)^{m+1} q^{(1-m)(2 m-1)}\left(1+q^{n+2 m}\right)\left(1-q^{2 m}\right)\left(-q^{2} ; q^{2}\right)_{2 m-1}
\end{aligned}
$$

\section{Main Results}

We will need to rewrite the Corollary 1 in terms of the Gaussian $q$-binomial coefficients and the $q$-Pochhammer symbol. Firstly we prove the first claim of the Corollary. It is stated as

$$
\sum_{k=0}^{m}\left\{\begin{array}{l}
4 m \\
4 k
\end{array}\right\}_{F} F_{n+4 m-4 k}=\frac{1}{2} F_{n+2 m} L_{2 m}\left((-1)^{m} \sum_{k=1}^{2 m-1} L_{k}^{2}+\sum_{k=1}^{2 m-1} L_{2 k}\right) .
$$

Before proving it, we convert the formula (3.1) into $q$-notation. The left hand side of it is:

$$
\sum_{k=0}^{m}\left\{\begin{array}{c}
4 m \\
4 k
\end{array}\right\}_{F} F_{n+4 m-4 k}
$$




$$
\begin{aligned}
= & \frac{\alpha^{4 m+n-1}}{(1-q)} \sum_{k=0}^{m}\left[\begin{array}{c}
4 m \\
4 k
\end{array}\right]_{q} \alpha^{-4 k+16 k m-16 k^{2}}\left(1-q^{n+4 m-4 k}\right) \\
= & \frac{\mathbf{i}^{n-1} q^{-\frac{n-1}{2}-2 m}}{(1-q)} \sum_{k=0}^{m}\left[\begin{array}{c}
4 m \\
4 k
\end{array}\right]_{q} q^{8 k^{2}+2 k-8 k m} \\
& -\frac{\mathbf{i}^{n-1} q^{\frac{n+1}{2}+2 m}}{(1-q)} \sum_{k=0}^{m}\left[\begin{array}{c}
4 m \\
4 k
\end{array}\right]_{q} q^{8 k^{2}-2 k-8 k m} .
\end{aligned}
$$

The right hand side of (3.1) is

$$
\begin{aligned}
\frac{1}{2} F_{n+2 m} L_{2 m}\left((-1)^{m} \sum_{k=1}^{2 m-1} L_{k}^{2}+\sum_{k=1}^{2 m-1} L_{2 k}\right)=\frac{1}{2}^{n-1} q^{-\frac{1}{2}\left(n+4 m^{2}+2 m-1\right)} \\
\times\left(1+q^{2 m}\right) \frac{\left(1-q^{n+2 m}\right)}{(1-q)}\left(\sum_{k=1}^{2 m-1}\left(1+q^{k}\right)^{2}+(-1)^{m} \sum_{k=1}^{2 m-1}\left(1+q^{2 k}\right)\right) .
\end{aligned}
$$

Thus we must show that

$$
\begin{gathered}
\left(q^{-\frac{n-1}{2}-2 m} \sum_{k=0}^{m}\left[\begin{array}{c}
4 m \\
4 k
\end{array}\right]_{q} q^{8 k^{2}+2 k-8 k m}-q^{\frac{n+1}{2}+2 m} \sum_{k=0}^{m}\left[\begin{array}{c}
4 m \\
4 k
\end{array}\right]_{q} q^{8 k^{2}-2 k-8 k m}\right) \\
\times \frac{\mathbf{i}^{n-1}}{1-q}= \\
\frac{1}{2} \mathbf{i}^{n-1} q^{-\frac{1}{2}\left(n+4 m^{2}+2 m-1\right)} \frac{\left(1+q^{2 m}\right)\left(1-q^{n+2 m}\right)}{(1-q)} \\
\times\left((-q ; q)_{2 m-1}^{2}+(-1)^{m}\left(-q^{2} ; q^{2}\right)_{2 m-1}\right) .
\end{gathered}
$$

Now we consider the first sums on the left hand side of the above equation and then compute it:

$$
\sum_{k=0}^{m}\left[\begin{array}{c}
4 m \\
4 k
\end{array}\right]_{q} q^{8 k^{2}+2 k-8 k m}=\frac{1}{4} \sum_{k=0}^{4 m}\left[\begin{array}{c}
4 m \\
k
\end{array}\right]_{q} q^{\left({ }^{k+1}\right)-2 m k}\left(1+(-1)^{k}+(-\mathbf{i})^{k}+\mathbf{i}^{k}\right),
$$

which, by Rothe's formula, equals

$$
\begin{aligned}
\frac{1}{4} \frac{\mathbf{i}^{n-1} q^{-\frac{1}{2}(4 m+n-1)}}{(1-q)}\left(\left(-q^{-2 m+1} ; q\right)_{4 m}\right. & +\left(q^{-2 m+1} ; q\right)_{4 m} \\
& \left.+\left(-\mathbf{i} q^{-2 m+1} ; q\right)_{4 m}+\left(\mathbf{i} q^{-2 m+1} ; q\right)_{4 m}\right),
\end{aligned}
$$

which, since $\left(q^{-2 m+1} ; q\right)_{4 m}=0$, gives us

$$
\sum_{k=0}^{m}\left[\begin{array}{c}
4 m \\
4 k
\end{array}\right]_{q} q^{8 k^{2}+2 k-8 k m}=\frac{1}{4} \frac{\mathbf{i}^{n-1} q^{-\frac{1}{2}(4 m+n-1)}}{(1-q)}
$$




$$
\times\left(\left(-q^{-2 m+1} ; q\right)_{4 m}+\left(-\mathbf{i} q^{-2 m+1} ; q\right)_{4 m}+\left(\mathbf{i} q^{-2 m+1} ; q\right)_{4 m}\right) .
$$

Now we consider the second sums on the left hand side of (3.2):

$$
\begin{aligned}
& \sum_{k=0}^{m}\left[\begin{array}{c}
4 m \\
4 k
\end{array}\right]_{q} q^{8 k^{2}-2 k-8 k m}=\frac{1}{4} \sum_{k=0}^{4 m}\left[\begin{array}{c}
4 m \\
4 k
\end{array}\right]_{q} q^{\left(\begin{array}{c}
k \\
2
\end{array}\right)-2 m k}\left(1+(-1)^{k}+(-\mathbf{i})^{k}+\mathbf{i}^{k}\right) \\
& \quad=\frac{\mathbf{i}^{n-1} q^{2 m+\frac{n+1}{2}}}{4(1-q)}\left(\left(-q^{-2 m} ; q\right)_{4 m}+\left(q^{-2 m} ; q\right)_{4 m}+\left(-\mathbf{i} q^{-2 m} ; q\right)_{4 m}+\left(\mathbf{i} q^{-2 m} ; q\right)_{4 m}\right),
\end{aligned}
$$

which, since $\left(q^{-2 m} ; q\right)_{4 m}=0$, equals

$$
\frac{\mathbf{i}^{n-1} q^{2 m+\frac{n+1}{2}}}{4(1-q)}\left(\left(-q^{-2 m} ; q\right)_{4 m}+\left(-i q^{-2 m} ; q\right)_{4 m}+\left(i q^{-2 m} ; q\right)_{4 m}\right) \text {. }
$$

Thus our original result takes the form:

$$
\begin{aligned}
& \frac{\mathbf{i}^{n-1}}{(1-q)}\left(q^{-\frac{n-1}{2}-2 m} \sum_{k=0}^{m}\left[\begin{array}{c}
4 m \\
4 k
\end{array}\right]_{q} q^{8 k^{2}+2 k-8 k m}-q^{\frac{n+1}{2}+2 m} \sum_{k=0}^{m}\left[\begin{array}{c}
4 m \\
4 k
\end{array}\right]_{q} q^{8 k^{2}-2 k-8 k m}\right) \\
= & \frac{\mathbf{i}^{n-1} q^{-2 m-\frac{n-1}{2}}}{4(1-q)}\left(\left(-q^{-2 m+1} ; q\right)_{4 m}+\left(-\mathbf{i} q^{-2 m+1} ; q\right)_{4 m}+\left(\mathbf{i} q^{-2 m+1} ; q\right)_{4 m}\right) \\
& -\frac{1}{4} \frac{\mathbf{i}^{n-1} q^{2 m+\frac{n+1}{2}}}{(1-q)}\left(\left(-q^{-2 m} ; q\right)_{4 m}+\left(-\mathbf{i} q^{-2 m} ; q\right)_{4 m}+\left(\mathbf{i} q^{-2 m} ; q\right)_{4 m}\right) \\
= & \frac{\mathbf{i}^{n-1}}{4(1-q)}\left(q^{-2 m-\frac{n-1}{2}}\left(-q^{-2 m+1} ; q\right)_{4 m}+q^{-2 m-\frac{n-1}{2}}\left(-\mathbf{i} q^{-2 m+1} ; q\right)_{4 m}\right. \\
& +q^{-2 m-\frac{n-1}{2}}\left(\mathbf{i} q^{-2 m+1} ; q\right)_{4 m}-q^{2 m+\frac{n+1}{2}}\left(-q^{-2 m} ; q\right)_{4 m} \\
& \left.-q^{2 m+\frac{n+1}{2}}\left(-\mathbf{i} q^{-2 m} ; q\right)_{4 m}-q^{2 m+\frac{n+1}{2}}\left(\mathbf{i} q^{-2 m} ; q\right)_{4 m}\right),
\end{aligned}
$$

which, by Lemma 2, equals

$$
\begin{aligned}
& \frac{1}{2} \mathbf{i}^{n-1} q^{-\frac{1}{2}\left(n+4 m^{2}+2 m-1\right)} \frac{\left(1-q^{n+2 m}\right)}{(1-q)} \\
& \quad \times\left(\frac{1}{\left(1+q^{2 m}\right)}(-q ; q)_{2 m}^{2}+(-1)^{m}\left(1+q^{2 m}\right)\left(-q^{2} ; q^{2}\right)_{2 m-1}\right) \\
& =\frac{1}{2} \mathbf{i}^{n-1} q^{-\frac{1}{2}\left(n+4 m^{2}+2 m-1\right)}\left(1+q^{2 m}\right) \frac{\left(1-q^{n+2 m}\right)}{(1-q)} \\
& \quad \times\left((-q ; q)_{2 m-1}^{2}+(-1)^{m}\left(-q^{2} ; q^{2}\right)_{2 m-1}\right),
\end{aligned}
$$

as claimed. 
The other claims in the Corollary 1 takes the following forms via the Gaussian $q$-binomial coefficients and the $q$-Pochhammer symbol. The second claim

$$
\begin{aligned}
\sum_{j=0}^{m-1}\left\{\begin{array}{c}
4 m \\
4 j+1
\end{array}\right\}_{F} F_{n+4 m-4 j-1} & \\
& =\frac{1}{2}\left((-1)^{m+1} F_{2 m+n} L_{2 m} \prod_{k=1}^{2 m-1} L_{k}^{2}+L_{2 m+n} F_{2 m} \prod_{k=1}^{2 m-1} L_{2 k}\right)
\end{aligned}
$$

is converted into the $q$-form as

$$
\begin{aligned}
\sum_{k=0}^{m-1} & {\left[\begin{array}{c}
4 m \\
4 k+1
\end{array}\right]_{q} q^{8 k^{2}+6 k-8 k m}\left(1-q^{n+4 m-4 k-1}\right) } \\
= & \frac{1}{2}(-1)^{m+1} q^{-2 m^{2}+3 m-1}\left((-1)^{m+1}\left(1-q^{2 m+n}\right)\left(1+q^{2 m}\right)(-q ; q)_{2 m-1}^{2}\right. \\
& \left.+\left(1+q^{2 m+n}\right)\left(1-q^{2 m}\right)\left(-q^{2} ; q^{2}\right)_{2 m-1}\right) .
\end{aligned}
$$

To prove the above claim, consider the left hand side of it:

$$
\begin{aligned}
& \sum_{k=0}^{m-1}\left[\begin{array}{c}
4 m \\
4 k+1
\end{array}\right]_{q} q^{8 k^{2}+6 k-8 k m}\left(1-q^{n+4 m-4 k-1}\right) \\
& =\sum_{k=0}^{m-1}\left[\begin{array}{c}
4 m \\
4 k+1
\end{array}\right]_{q} q^{8 k^{2}+6 k-8 k m}-q^{n+4 m-1} \sum_{k=0}^{m-1}\left[\begin{array}{c}
4 m \\
4 k+1
\end{array}\right]_{q} q^{8 k^{2}+2 k-8 k m} \\
& =\frac{1}{4} \sum_{k=0}^{4 m-4}\left[\begin{array}{c}
4 m \\
k+1
\end{array}\right]_{q}^{q^{\frac{1}{2} k(k-4 m+3)}\left(1+(-1)^{k}+\mathbf{i}^{k}+(-\mathbf{i})^{k}\right)} \\
& -\frac{1}{4} q^{n+4 m-1} \sum_{k=0}^{4 m-4}\left[\begin{array}{c}
4 m \\
k+1
\end{array}\right]_{q} q^{\frac{1}{2} k(k-4 m+1)}\left(1+(-1)^{k}+\mathbf{i}^{k}+(-\mathbf{i})^{k}\right) .
\end{aligned}
$$

Let $f(k)=q^{\frac{1}{2} k(k-4 m+3)}\left(1+(-1)^{k}+\mathbf{i}^{k}+(-\mathbf{i})^{k}\right)$. Since $f(-1)=0$, for the first sum in the above equation, we can write

$$
\begin{aligned}
& \sum_{k=0}^{4 m-4}\left[\begin{array}{c}
4 m \\
4 k+1
\end{array}\right]_{q} q^{\frac{1}{2} k(k-4 m+3)}\left(1+(-1)^{k}+\mathbf{i}^{k}+(-\mathbf{i})^{k}\right) \\
& =\sum_{k=0}^{4 m-4}\left[\begin{array}{c}
4 m \\
k+1
\end{array}\right]_{q} f(k)=\sum_{k=1}^{4 m-3}\left[\begin{array}{c}
4 m \\
k
\end{array}\right]_{q} f(k-1)
\end{aligned}
$$




$$
\begin{aligned}
& =\sum_{k=0}^{4 m-3}\left[\begin{array}{c}
4 m \\
k
\end{array}\right]_{q} f(k-1)-f(-1)=\sum_{k=0}^{4 m-3}\left[\begin{array}{c}
4 m \\
k
\end{array}\right]_{q} f(k-1) \\
& =\sum_{k=0}^{4 m-3}\left[\begin{array}{c}
4 m \\
k
\end{array}\right]_{q} q^{\frac{1}{2}(k-1)(k-4 m+2)}\left(1+(-1)^{k-1}+\mathbf{i}^{k-1}+(-\mathbf{i})^{k-1}\right) .
\end{aligned}
$$

Similarly for the second sum in the same equation, we write

$$
\begin{aligned}
& \sum_{k=0}^{4 m-4}\left[\begin{array}{c}
4 m \\
k+1
\end{array}\right]_{q} q^{\frac{1}{2} k(k-4 m+1)}\left(1+(-1)^{k}+\mathbf{i}^{k}+(-\mathbf{i})^{k}\right) \\
& =\sum_{k=0}^{4 m-3}\left[\begin{array}{c}
4 m \\
k
\end{array}\right]_{q}^{q^{\frac{1}{2}(k-1)(k-4 m)}\left(1+(-1)^{k-1}+\mathbf{i}^{k-1}+(-\mathbf{i})^{k-1}\right) .}
\end{aligned}
$$

Consequently we get

$$
\begin{aligned}
& \sum_{k=0}^{m-1}\left[\begin{array}{c}
4 m \\
4 k+1
\end{array}\right]_{q} q^{8 k^{2}+6 k-8 k m}\left(1-q^{n+4 m-4 k-1}\right) \\
& =\frac{1}{4} q^{2 m-1} \sum_{k=0}^{4 m-3}\left[\begin{array}{c}
4 m \\
k
\end{array}\right]_{q} q^{\left(\begin{array}{c}
k \\
2
\end{array}\right)+(1-2 m) k}\left(1+(-1)^{k-1}+\mathbf{i}^{k-1}+(-\mathbf{i})^{k-1}\right) \\
& \quad-\frac{1}{4} q^{n+6 m-1} \sum_{k=0}^{4 m-3}\left[\begin{array}{c}
4 m \\
k
\end{array}\right]_{q} q^{\left(\begin{array}{c}
k \\
2
\end{array}\right)-2 m k}\left(1+(-1)^{k-1}+\mathbf{i}^{k-1}+(-\mathbf{i})^{k-1}\right) .
\end{aligned}
$$

Let

$$
\begin{aligned}
& A=\frac{q^{1-2 m}\left(1-q^{4 m}\right)\left(1-q^{4 m-1}\right)}{\left(q^{2}-1\right)(q-1)}-\frac{\left(1-q^{4 m}\right)}{q-1}+q^{2 m}, \\
& B=\frac{q^{1-2 m}\left(1-q^{4 m}\right)\left(1-q^{4 m-1}\right)}{\left(1-q^{2}\right)(1-q)}-\frac{\left(1-q^{4 m}\right)}{(1-q)}+q^{2 m}, \\
& C=-\frac{q^{1-2 m}\left(1-q^{4 m}\right)\left(1-q^{4 m-1}\right)}{\left(1-q^{2}\right)(1-q)}-\mathbf{i} \frac{\left(1-q^{4 m}\right)}{(1-q)}+q^{2 m}, \\
& D=-\frac{q^{1-2 m}\left(1-q^{4 m}\right)\left(1-q^{4 m-1}\right)}{\left(1-q^{2}\right)(1-q)}+\mathbf{i} \frac{\left(1-q^{4 m}\right)}{(1-q)}+q^{2 m},
\end{aligned}
$$

and

$$
\begin{aligned}
& A_{1}=\frac{q^{3-6 m}\left(1-q^{4 m}\right)\left(1-q^{4 m-1}\right)}{\left(1-q^{2}\right)(1-q)}+\frac{q^{1-4 m}\left(1-q^{4 m}\right)}{1-q}+q^{-2 m} \\
& B_{1}=\frac{q^{3-6 m}\left(1-q^{4 m}\right)\left(1-q^{4 m-1}\right)}{\left(1-q^{2}\right)(1-q)}-\frac{q^{1-4 m}\left(1-q^{4 m}\right)}{1-q}+q^{-2 m}
\end{aligned}
$$




$$
\begin{aligned}
& C_{1}=-\frac{q^{3-6 m}\left(1-q^{4 m}\right)\left(1-q^{4 m-1}\right)}{\left(1-q^{2}\right)(1-q)}-\mathbf{i} \frac{q^{1-4 m}\left(1-q^{4 m}\right)}{(1-q)}+q^{-2 m} \\
& D_{1}=-\frac{q^{3-6 m}\left(1-q^{4 m}\right)\left(1-q^{4 m-1}\right)}{\left(1-q^{2}\right)(1-q)}+\mathbf{i} \frac{q^{1-4 m}\left(1-q^{4 m}\right)}{(1-q)}+q^{-2 m} .
\end{aligned}
$$

Then clearly $-A+B+\mathbf{i} C-\mathbf{i} D=0$ and $-A_{1}+B_{1}+\mathbf{i} C_{1}-\mathbf{i} D_{1}=0$. Thus

$$
\begin{aligned}
& \frac{1}{4} q^{2 m-1} \sum_{k=0}^{4 m-3}\left[\begin{array}{c}
4 m \\
k
\end{array}\right]_{q} q^{\left(\begin{array}{l}
k \\
2
\end{array}\right)+(1-2 m) k}\left(1+(-1)^{k-1}+\mathbf{i}^{k-1}+(-\mathbf{i})^{k-1}\right) \\
& -\frac{1}{4} q^{n+6 m-1} \sum_{k=0}^{4 m-3}\left[\begin{array}{c}
4 m \\
k
\end{array}\right]_{q} q^{\left(\begin{array}{l}
k \\
2
\end{array}\right)-2 m k}\left(1+(-1)^{k-1}+\mathbf{i}^{k-1}+(-\mathbf{i})^{k-1}\right) \\
& =\frac{1}{4} q^{2 m-1}\left(\left(\left(-q^{1-2 m} ; q\right)_{4 m}-A\right)-\left(\left(q^{1-2 m} ; q\right)_{4 m}-B\right)\right. \\
& \left.\quad-\mathbf{i}\left(\left(-\mathbf{i} q^{1-2 m} ; q\right)_{4 m}-C\right)+\mathbf{i}\left(\left(\mathbf{i} q^{1-2 m} ; q\right)_{4 m}-D\right)\right) \\
& \quad-\frac{1}{4} q^{n+6 m-1}\left(\left(\left(-q^{-2 m} ; q\right)_{4 m}-A_{1}\right)-\left(\left(q^{-2 m} ; q\right)_{4 m}-B_{1}\right)\right. \\
& \left.\quad-\mathbf{i}\left(\left(-\mathbf{i} q^{-2 m} ; q\right)_{4 m}-C_{1}\right)+\mathbf{i}\left(\left(\mathbf{i} q^{-2 m} ; q\right)_{4 m}-D_{1}\right)\right),
\end{aligned}
$$

which, since $\left(q^{1-2 m} ; q\right)_{4 m}=0$ and $\left(q^{-2 m} ; q\right)_{4 m}=0$, gives us

$$
\begin{aligned}
= & \frac{1}{4} q^{2 m-1}\left(\left(-q^{1-2 m} ; q\right)_{4 m}-\mathbf{i}\left(-\mathbf{i} q^{1-2 m} ; q\right)_{4 m}+\mathbf{i}\left(\mathbf{i} q^{1-2 m} ; q\right)_{4 m}\right) \\
& -\frac{1}{4} q^{n+6 m-1}\left(\left(-q^{-2 m} ; q\right)_{4 m}-\mathbf{i}\left(-\mathbf{i} q^{-2 m} ; q\right)_{4 m}+\mathbf{i}\left(\mathbf{i} q^{-2 m} ; q\right)_{4 m}\right) .
\end{aligned}
$$

By Lemma 3, we write the last equation as

$$
\begin{aligned}
=\frac{\left(1-q^{n+2 m}\right)\left(1+q^{2 m}\right)}{2 q^{(m-1)(2 m-1)}} \prod_{k=1}^{2 m-1}\left(1+q^{k}\right)^{2}+ \\
\quad(-1)^{m+1} \frac{\left(1+q^{n+2 m}\right)\left(1-q^{2 m}\right)}{2 q^{(m-1)(2 m-1)}} \prod_{k=1}^{2 m-1}\left(1+q^{2 k}\right),
\end{aligned}
$$

as claimed.

As we state as before, if one have the Corollary 1, then the claims of Conjecture could be easily derived. As an example, since we have first two claims of Corollary 1 , we can obtain the first claim of Conjecture :

$$
\sigma_{1}(n)+\sigma_{2}(n)=\frac{1}{2} F_{2 m+n} L_{2 m}\left((-1)^{m} \prod_{k=1}^{2 m-1} L_{k}^{2}+\prod_{k=1}^{2 m-1} L_{2 k}\right)
$$




$$
\begin{aligned}
& +\frac{1}{2}\left((-1)^{m+1} F_{2 m+n} L_{2 m} \prod_{k=1}^{2 m-1} L_{k}^{2}+L_{2 m+n} F_{2 m} \prod_{k=1}^{2 m-1} L_{2 k}\right) \\
= & \frac{1}{2}\left(F_{2 m+n} L_{2 m}+L_{2 m+n} F_{2 m}\right) \prod_{k=1}^{2 m-1} L_{2 k} \\
= & F_{4 m+n} \prod_{k=1}^{2 m-1} L_{2 k},
\end{aligned}
$$

as claimed in the Conjecture.

After that we only state the other claims of Corollary 1 in $q$-notation and leave the proofs of them to the readers. They are similarly proved as we did previously.

The claims (iii) and (iv) in Corollary 1 take the following forms in $q$-notation:

$$
\begin{aligned}
& \sum_{k=0}^{m-1}\left[\begin{array}{c}
4 m \\
4 k+2
\end{array}\right]_{q} q^{2 k(4 k-4 m+5)}\left(1-q^{n+4 m-4 k-2}\right) \\
& =\frac{1}{2}(-1)^{m+1} q^{-(m-1)(2 m-3)}\left(1-q^{2 m+n}\right)\left(1+q^{2 m}\right) \\
& \quad \times\left((-1)^{m+1}(-q ; q)_{2 m-1}^{2}+\left(-q^{2} ; q^{2}\right)_{2 m-1}\right)
\end{aligned}
$$

and

$$
\begin{aligned}
& \sum_{k=0}^{m-1}\left[\begin{array}{c}
4 m \\
4 k+3
\end{array}\right]_{q} q^{2 k(4 k-4 m+7)}\left(1-q^{n+4 m-4 k-3}\right) \\
& =\frac{1}{2}(-1)^{m} q^{-2 m^{2}+7 m-6}\left((-1)^{m}\left(1-q^{2 m+n}\right)\left(1+q^{2 m}\right)(-q ; q)_{2 m-1}^{2}\right. \\
& \left.\quad+\left(1+q^{2 m+n}\right)\left(1-q^{2 m}\right)\left(-q^{2} ; q^{2}\right)_{2 m-1}\right) .
\end{aligned}
$$

By following the proof way of the claims (i) and (ii), the above claims could be proved similarly.

\section{REFERENCES}

[1] G. E. Andrews, R. Askey, and R. Roy, Special functions. Paperback ed, ser. Encyclopedia of Mathematics and Its Applications. Cambridge: Cambridge University Press, 2000, vol. 71.

[2] H. W. Gould, "The bracket function and Fontené-Ward generalized binomial coefficients with application to Fibonomial coefficients," Fibonacci Q., vol. 7, pp. 23-40, 1969.

[3] V. E. Hoggatt, "Fibonacci numbers and generalized binomial coefficients," Fibonacci Q., vol. 5, pp. 383-400, 1967.

[4] E. Kilic, I. Akkus, and H. Ohtsuka, "Some generalized Fibonomial sums related with the Gaussian q-binomial sums," Bull. Math. Soc. Sci. Math. Roum., Nouv. Sér., vol. 55, no. 1, pp. 51-61, 2012.

[5] E. Kiliç, “The generalized Fibonomial matrix," Eur. J. Comb., vol. 31, no. 1, pp. 193-209, 2010. 
[6] E. Kiliç, H. Prodinger, I. Akkus, and H. Ohtsuka, "Formulas for fibonomial sums with generalized Fibonacci and Lucas coefficients," Fibonacci Q., vol. 49, no. 4, pp. 320-329, 2011.

[7] D. Marques and P. Trojovský, "On some new sums of Fibonomial coefficients," Fibonacci Q., vol. 50, no. 2, pp. 155-162, 2012.

\section{Author's address}

\section{Emrah Kılıç}

TOBB Economics and Technology University, Mathematics Department, 06560 Ankara, Turkey E-mail address: ekilic@etu.edu.tr 